\title{
Mobile Gaming with Indirect Sensor Control
}

\author{
Daniel Böhrs, Dirk Wenig, and Rainer Malaka \\ Research Group Digital Media, TZI, \\ University of Bremen, Bibliothekstr. 1, 28359 Bremen, Germany
}

\begin{abstract}
The rapid growth of the mobile gaming market and the steadily improved hardware of mobile phones enable developers to create complex and extensive 3D games on mobile phones. While most current casual games have simple interfaces with few buttons, 3D games require new control interfaces to providing sufficient control options without limiting the field of view on the screen. This is important to improve the user experience. Within this work new ideas based on the use of the accelerometer as indirect control mechanism are presented. The accelerometer is used to switch between different interaction layers, which are also different game views for the player. Combined with this concept a buttonless touch area interface is used. We are planning to evaluate the ideas with a 3D game prototype running on Android devices.
\end{abstract}

Keywords: mobile gaming, sensors, mobile devices, labyrinth game.

\section{Introduction}

Controls for mobile phones are mostly touch screen and sensor interfaces. Currently this results in an adaption of conventional hardware elements from computers or consoles to build similar game controls on mobile phones. In games with various interaction possibilities, problems can occur while trying to map all needed controls on the display because the field of view can be limited substantially. Another approach is the use of sensors like the accelerometer, which is built into nearly all modern devices. This technique is especially popular in the racing game genre. A problem is that turning the device to the sides or shaking it can cause a lack of the overview. Both concepts limit the field of view in different ways. In order to tackle this challenge, we present the concept of an adapted combination of a accelerometer and a touch interface. The touch controls are integrated as a buttonless interface by using touch areas while the accelerometer is used as an indirect control mechanism. It enables the player to switch not only between different views, but also between controls. The overall result should be a clear interface with no disruptive objects on the screen and providing as much control as possible.

\section{Related Work}

Chu, $\mathrm{K}$ et al. [1] showed that mobile games on touch devices generally have a lack of comfort in the control system because of not existing hardware controls. New approaches must be found or existing ones have to be improved. Chui Yin Wong et al. [3] published study results demonstrating advantages of optimized touch interfaces in 
comparison to hardware controls. For example, the best scores while playing were arrived on touch interface and the amount of miss clicks was very low. An alternative approach is the use of the accelerometer which is already well established. This solution is mentioned by Kevin Browne et al. [2]: They used a touch based interface, one with an accelerometer and a gesture based system within a 2D scroll shooter game. The accelerometer interface was preferred by the testers and led to the best results in experience measurements. The use of accelerometers in 3D game is demonstrated by Fadi Chehimi and Paul Coulton [4] and is analogical to 2D games while using more axes for calculating movements within the game. The use of the accelerometer was rated mostly positive. Paul Gilbertson et al. [5] used the same approach but realized the game tunnel run without any buttons. The evaluation showed that players favor this type of control interface and the user experience ratings increase.

\section{Concept}

The developed prototype is a time based ball-through-labyrinth game with two different perspectives. Within the labyrinth corridors are objects, which must be passed by the user. The ball moves forward continuously without users control. One view is the topdown view, which is active if the device is placed horizontally (fig. 10. In this view the player can rotate left or right. The other view is a third-person view which allows the player to navigate around obstacles inside a corridor. In this view the player shifts the ball to the left or right.

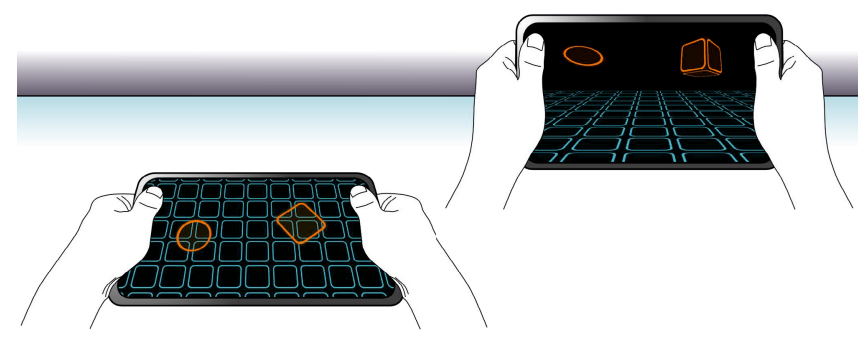

Fig. 1. Concept of a pitch gesture

The player is not forced to switch the view because he is able to see the map and the obstacles in both views. But the ability of swiftly moving around an obstacle by translating is a huge benefit which should motivate the players to switch the views by themselfs. This game is the basis for the idea of buttonless interaction in games with different control layers and views switched with the accelerometer. Within the concept multiple controls can be applied to smartphones without obstructing the players view and reducing screen-space by placing numerous visible buttons. 


\section{Control Concepts}

Overall three concepts were implemented as parts of this work. A touch area and sensor interface, a touch area interface and a traditional touch button interface. The last one is used as control compare with the evaluation results of the other two approaches.

\subsection{Touch Areas with Accelerometer Support}

The first concept provides no visible objects on the touch screen (fig. 2). It is is divided vertically in two areas. Pushing the left side will trigger an action to the left and similar behavior is triggered on the right side. In the top view rotation is done by pushing the areas while in the third person view pushing leads to translations to move around obstacles. The switching between the two views is done by pitching the device. Holding it horizontally brings up the top view while holding it obliquely will switch to the third person view. The result is a clean screen and an intuitive way to switch the views.

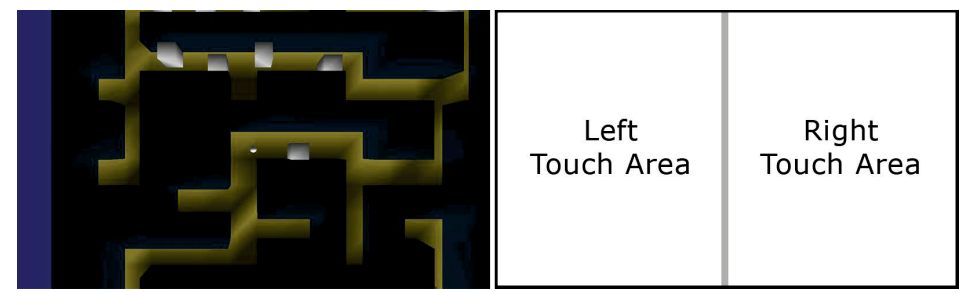

Fig. 2. Touch areas with accelerometer support

\subsection{Touch Areas without Accelerometer Support}

The second concept is based on the first one but without sensor interactions. Instead of switching the views by tending, another touch area is inserted at the bottom (fig. (3). This concepts reduces the interaction to touch controls without limiting the field of view.
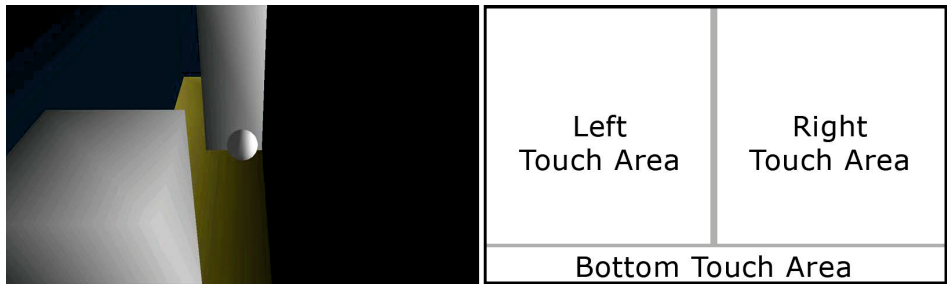

Fig. 3. Touch areas without accelerometer support 


\subsection{Traditional Touch Button Controls}

The third concept is based on the known techniques by placing visible buttons on the screen to allow interaction (fig. (4). The number of buttons for this concept is 6: Two buttons to rotate, two to translate and two to switch the view. The buttons for switching the view are toggle buttons. The translation and view switching can be done in all views while the rotation is only possible in the top view.

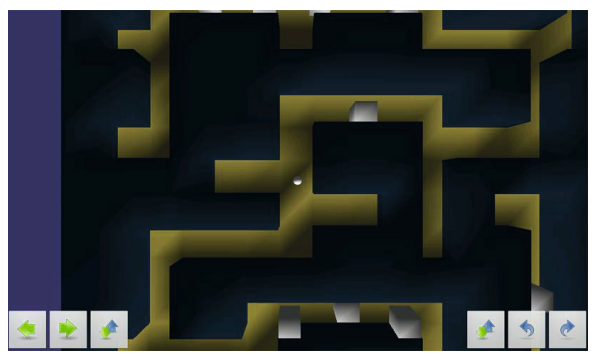

Fig. 4. Traditional touch button controls

\section{Conclusion and Future Work}

Currently we are planning an evaluation of the three concepts. Therefore, the presented prototype logs the interactions and sends them to a database for evaluation. At the end of the game a questionnaire is presented to get feedback about the users experience. The evaluation will investigate the advantages and disadvantages of the three control concepts and tests if the sensor support and the touch area concept could be approved as working. Every participant will play all three modes in random order. Future studies could adapt the concept for more complex games like shooters or role playing games.

\section{References}

1. Chu, K., Wong, C.Y.: Mobile input devices for gaming experience. In: User Science and Engineering (i-USEr), 2011 International Conference, November 29-December 1, pp. 83-88 (2011)

2. Browne, K., Anand, C.: An empirical evaluation of user interfaces for a mobile video game. Entertainment Computing 3(1), 1-10 (2012) ISSN 1875-9521

3. Wong, C.Y., Chu, K., Khong, C.W., Lim, T.Y.: Evaluating playability on haptic user interface for mobile gaming. In: Information Technology (ITSim), 2010 International Symposium, June 15-17, vol. 3, pp. 1093-1098 (2010)

4. Chehimi, F., Coulton, P.: Motion controlled mobile 3D multiplayer gaming. In: Proceedings of the 2008 International Conference on Advances in Computer Entertainment Technology (ACE 2008), pp. 267-270. ACM (2008)

5. Gilbertson, P., Coulton, P., Chehimi, F., Vajk, T.: Using tilt as an interface to control no-button mobile games. Comput. Entertain. 6(3), Article 38, 38:1-38:13 (2008) 